\title{
Prenatal diagnosis by FISH of a 22q11 deletion in two families
}

Marie-France Portnoï, Nicole Joyé, Marie Gonzales, Suzanne Demczuk, Laurent Fermont, Gilles Gaillard, Guy Bercau, Geneviève Morlier, Jean-Louis Taillemite

\begin{abstract}
We report on prenatal diagnosis by FISH of a sporadic 22 q11 deletion associated with DiGeorge syndrome (DGS) in two fetuses after an obstetric ultrasonographic examination detected cardiac anomalies, an interrupted aortic arch in case 1 and tetralogy of Fallot in case 2. The parents decided to terminate the pregnancies. At necropsy, fetal examination showed characteristic facial dysmorphism associated with congenital malformations, confirming full DGS in both fetuses. In addition to the 22q11 deletion, trisomy $X$ was found in the second fetus and a reciprocal balanced translocation $t(11 ; 22)(q 23 ; q 11)$ was found in the clinically normal father of case 1 . These findings highlight the importance of performing traditional cytogenetic analysis and FISH in pregnancies with a high risk of having a deletion.

(f Med Genet 1998;35:165-168)
\end{abstract}

Pathologique et de

Cytogénétique, Hôpital

Saint-Antoine, 75012

Paris, France

M-F Portnoï

N Joyé

$M$ Gonzales

G Morlier

J-L Taillemite

Cytogenetics, Prenatal Diagnosis, Montreal Children's Hospital, Montreal, Quebec, Canada

S Demczuk

Unité d'Explorations

Cardiologiques,

Institut de

Puériculture de Paris,

75014 Paris, France

L Fermont

Service de Gynécologie et Obstétrique, Hôpital Saint-Antoine, 75012

Paris, France

G Gaillard

Service de Gynécologie et Obstétrique, Hôpital Notre-Dame de

Bon-Secours, 75014

Paris, France

G Bercau

Correspondence to:

Dr Portnoï.

Received 8 April 1997 Revised version accepted for publication 30 September 1997
Keywords: DiGeorge syndrome; chromosome 22q11; FISH; microdeletion

DiGeorge syndrome (DGS), an aetiologically heterogeneous developmental field defect of the third and fourth pharyngeal pouches, is characterised by hypoplasia or aplasia of the thymus and parathyroid glands, a conotruncal heart defect, and varying craniofacial dysmorphism.' DGS is frequently associated with a chromosome 22 abnormality. Unbalanced translocations which result in monosomy of 22pter-22q11 and interstitial deletions have mapped the DGS region to $22 \mathrm{q} 11.2 .^{23}$ Molecular studies using probes for various loci in the $22 \mathrm{q} 11$ region have detected submicroscopic deletions in more than $90 \%$ of DGS cases without visible cytogenetic abnormalities. ${ }^{46}$

Deletions within $22 \mathrm{q} 11$ are associated with a wide variety of birth defects embraced by the acronym CATCH 22, ${ }^{7}$ including Sprintzen syndrome (velocardiofacial syndrome), conotruncal congenital heart disease, and DGS at the more severe end of the clinical spectrum. This haploinsufficiency of $22 \mathrm{q} 11$ is a relatively frequent cause of birth defect (1/5000 live births) ${ }^{8}$ Fluorescence in situ hybridisation (FISH) using unique sequence DNA probes is an efficient, quick, and direct method for detection of $22 \mathrm{q} 11$ microdeletions.

We report on prenatal diagnosis by FISH of a 22q11 deletion associated with DGS in two families. Both of these cases are sporadic. Moreover, in the first case, a reciprocal balanced translocation $\mathrm{t}(11 ; 22)(\mathrm{q} 23 ; \mathrm{q} 11)$ was shown in the father's karyotype; in the second case trisomy $\mathrm{X}$ was associated with a $22 \mathrm{q} 11$ deletion in the fetus.

\section{Materials and methods}

KARYOTYPING AND FISH

Fetal blood samples were obtained for karyotype analysis and FISH. Cells were harvested from cultures of phytohaemagglutinin stimulated lymphocytes and spread onto slides for the production of $\mathrm{G}$ banded or $\mathrm{R}$ banded chromosomes.

FISH of metaphase chromosomes using digoxigenin labelled cosmid probes D22S75 (N25, ONCOR) from the DGS chromosome region was carried out basically according to Pinkel et al. ${ }^{9}$ This probe was premixed with a control cosmid (D22S39) in 22q13.3 facilitating chromosome identification.

\section{Case reports}

CASE 1

A 31 year old, nulliparous woman, who had had a previous miscarriage at 8 weeks of gestation, was seen for fetal echocardiography at 23 weeks of gestation after an obstetric ultrasonographic examination detected cardiac anomalies and absence of the thymic shadow. Fetal echocardiography showed an interrupted aortic arch (IAA) associated with a membranous ventricular septal defect. The possibility of DGS was discussed because of its strong association with IAA and absence of the thymus. A fetal blood sample was obtained for karyotype determination. A 46,XX karyotype with a 22q11 deletion detected by FISH was found, consistent with the DG phenotype and provided a prenatal diagnosis of DGS (fig 1). The serum calcium level was determined and showed hypocalcaemia $(66 \mathrm{mg} / \mathrm{l}$, normal fetal

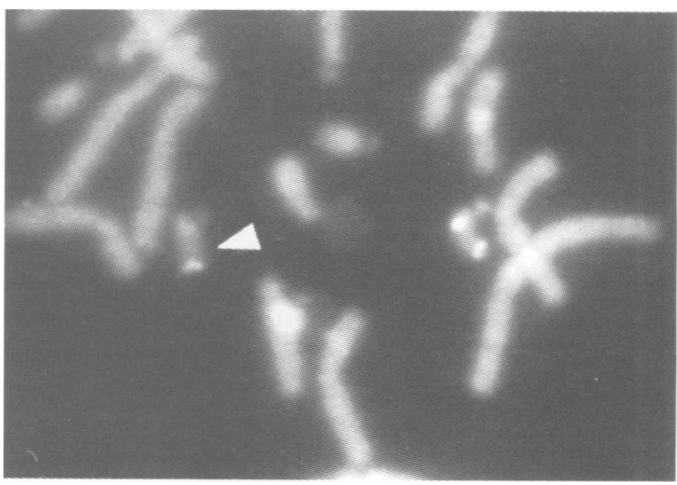

Figure 1 Partial metaphase spread showing lack of signal on one of the chromosomes 22. The deleted chromosome 22 with distal $q$ arm marker present, but without DiGeorge probe signal is indicated by an arrowhead. 


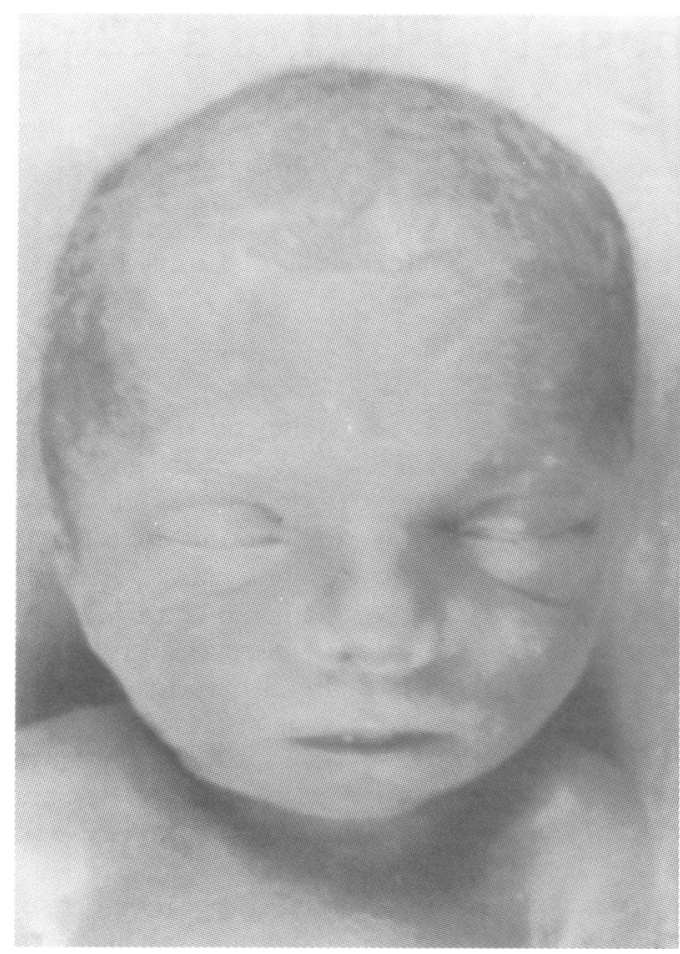

Figure 2 Fetus 1.
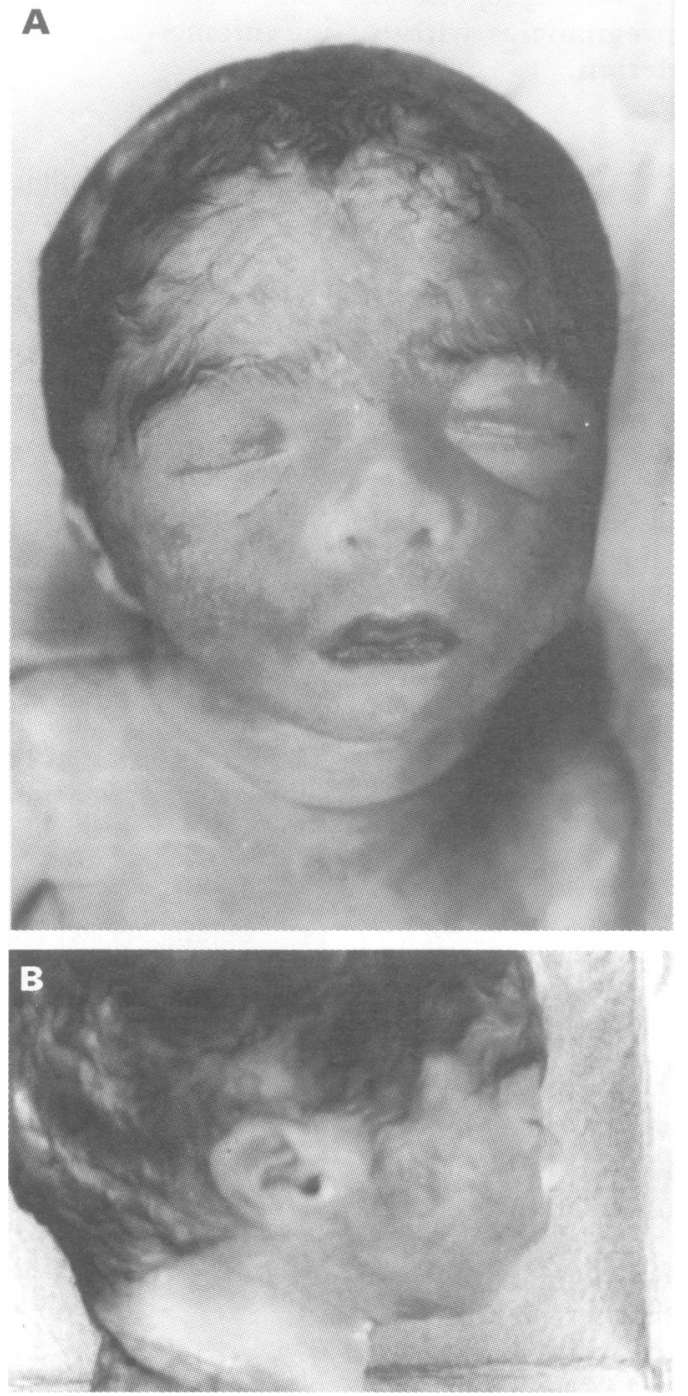

Figure 3 Fetus 2.

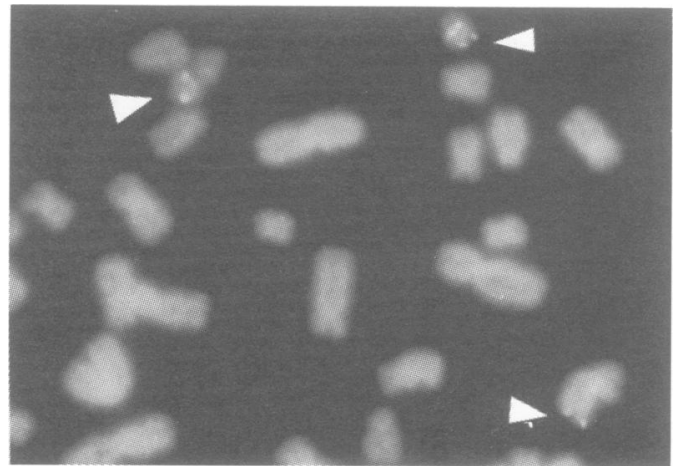

Figure 4 Partial metaphase spread of the father of case 1 after FISH showing a fluorescent signal on both

chromosome 22 homologues at 22 11.2 with probe D22S75 (ONCOR) and at 11q23 on der(11) and on normal chromosome 22 with probe D22S39.

levels $90.2 \pm 8 \mathrm{mg} / \mathrm{l})$. $\mathrm{T}$ cell subpopulation studies were not performed. The parents were informed about the risks and decided to terminate the pregnancy.

At necropsy, fetal examination showed facial dysmorphism with hypertelorism, a square nasal tip, a small, recessed chin, and a small hypoplastic thymus (fig 2). Cardiac examination confirmed the ultrasonographic findings. The aortic arch ended after the left common carotid artery, consistent with type B interruption, and was associated with a ventricular septal defect.

Neither parent had any evidence of a 22q11 deletion by FISH. The mother had normal chromosomes and the father was found to have a balanced reciprocal translocation involving chromosome $22, \mathrm{t}(11 ; 22)$ (q23; 111$)$.

\section{CASE 2}

Fetal blood was obtained for karyotype analysis from a fetus whose mother and father were 27 and 40 years of age, respectively. Prenatal ultrasound examination at 23 weeks showed tetralogy of Fallot (TF).

The fetus was found to have a $47, \mathrm{XXX}$ karyotype and a 22q11 deletion detected by FISH. Neither the serum calcium level nor the lymphocyte population was evaluated in this case. The TF associated with a $47, \mathrm{XXX}$ karyotype and a 22q11 deletion had obvious implications for genetic counselling and risk assessment. Following counselling the pregnancy was terminated.

At necropsy, the fetus had facial dysmorphic features including a broad nose, downward slanting palpebral fissures with hypertelorism, a small mouth, microstomia, micrognathia, rounded, posteriorly rotated ears with absent ear lobes, and facial hypertrichosis (fig 3). TF was associated with a complete absence of the thymus, vertebral anomalies, and a left talipes equinovarus deformity.

The parental karyotypes were both normal. Deletion 22q11 was excluded by FISH.

\section{Discussion}

Very few data are available concerning the prognosis for fetuses diagnosed prenatally as having a 22q11 deletion. To the best of our knowledge only three cases of prenatally 
diagnosed DGS with a 22q11 deletion have been published.

Van Hemel et $a l^{10}$ described a familial case illustrating the variable clinical expression of the chromosome 22q11 deletion. A 22q11 deletion was found in a child who died two weeks after birth with symptoms of full DGS and truncus arteriosus. This deletion was detected in the physically normal father who had mild learning disabilities and a tendency to depression, as well as in a subsequent pregnancy. Ultrasound studies did not show cardiac or other anomalies in the latter fetus. At birth the boy developed hypocalcaemia and had a moderate $\mathrm{T}$ cell deficit. Echocardiography showed a right sided aortic arch without intracardiac anomalies.

Puder et $a l^{11}$ reported a case of prenatally diagnosed IAA, which led to the detection of segmental monosomy of chromosome 22q11 by FISH in the fetus and in his mother, who had a small ventricular septal defect.

The first case of prenatal detection of a fetus with an interrupted aortic arch and a 22q11 deletion, in the absence of a family history, was reported by Davidson et al. ${ }^{12}$ The mother was also found to carry the deletion; she had no cardiac abnormality but was mildly retarded. The pregnancy continued and the prenatal diagnosis was helpful for planning postnatal care. In this family and in others, the deletion was transmitted from the mother to her more severely affected child. The range of phenotypes associated with the $22 \mathrm{q} 11$ deletion complicates genetic counselling. Moreover, so far there has been no experience with the progeny of severely affected subjects who nowadays survive after heart surgery.

Both of our cases were referred for cordocentesis and fetal blood karyotype analysis in the 23rd week of pregnancy because of detection by ultrasound examination of a cardiac anomaly, an IAA associated with thymic aplasia in the first case and TF in the second.

IAA, truncus arteriosus, unusual cardiovascular lesions, and TF are congenital conotruncal heart diseases commonly found in DGS. ${ }^{13}$ The results of recent molecular studies in patients with $T F$, including isolated $T F$ and syndromic cases, suggest that isolated TF is not associated with a $22 \mathrm{q} 11$ deletion, while hemizygosity for $22 \mathrm{q} 11$ is present in patients with TF associated with additional cardiovascular or non-cardiac anomalies. ${ }^{14} 15$ Therefore, detection of these cardiac diseases during pregnancy should prompt the investigation of characteristic abnormalities associated with DGS. These associated anomalies are, however, difficult to detect by prenatal diagnosis.

The discovery during pregnancy of a conotruncal heart defect associated with a 22q11 deletion might indicate a severe form of this syndrome, which is known to show great phenotypic variability. The most severely affected patients have serious, life threatening heart defects and die neonatally. The least severely affected patients have only mild facial anomalies and some developmental delay, but no heart defects. ${ }^{16}$ However, most of the patients with DGS who have survived infancy have been mildly to moderately retarded.

The presence of a deletion associated with conotruncal heart disease is an important point which has obvious implications for genetic counselling. In both of our cases, necropsy confirmed that the fetuses were severely affected and had multiple congenital anomalies. This study, the first to our knowledge that describes fetal examination of DGS with a $22 \mathrm{q} 11$ deletion, has shown that facial dysmorphism is present and recognisable in the fetus and is very similar to that of the newborn.

Furthermore, in our report, karyotype analysis showed a second chromosomal abnormality. In the first case, the father was found to have a balanced translocation $t(11 ; 22)(q 23 ; q 11)$. FISH analysis showed D22S75 to be present on the derivative 22 translocation chromosome and to be located centromeric to the translocation breakpoint in $22 \mathrm{q} 11$ (fig 4). The presence of a $22 \mathrm{q} 11$ deletion in the offspring of $t(11 ; 22)$ carriers has not been reported previously. An attractive hypothesis is that this recurrent translocation in the father may have played a role in the existence of the deletion in the fetus. Structural characteristics of the DNA in the 22q11 region, especially the presence of low copy repeat elements, would favour interstitial deletion with more or less precise breakpoint sites. ${ }^{16}{ }^{17}$ However, the role of chance cannot be excluded. Published data have shown a higher frequency of deletions of maternal origin $(70 \%) .{ }^{18}$ In the present case, determination of the parental origin of the deleted chromosome 22 in the fetus was attempted, but was not informative with the polymorphic marker used. Further endeavours to obtain other polymorphic markers mapping to the DGS deleted region and to continue the investigation into this family are in progress.

When neither parent has a deletion, it is expected that there will be a low risk of having a further child with a 22q11 deletion since gonadal mosaicism cannot be ruled out. In this family, an early first trimester prenatal diagnosis is relevant because of the risk of inheriting an unbalanced translocation derivative in the offspring.

In the second case a triple $\mathrm{X}$ karyotype was found and the association of triple $X$ with DGS has never previously been reported either. However, trisomy $\mathrm{X}$ is a relatively common aneuploidy and we suggest that this association is likely to be a chance event. The finding of this anomaly might be an indication for prenatal diagnosis in a subsequent pregnancy.

These findings highlight the importance of performing traditional cytogenetic analysis and FISH for suspected DGS during pregnancy when ultrasound studies show a conotruncal cardiac defect. In these cases prenatal diagnosis of a molecular deletion in the DGS critical region of chromosome 22 suggests the existence of associated anomalies and risks of developmental delay. However, counselling remains difficult in view of the clinical variabil- 
ity described in DGS, where the phenotype cannot be accurately predicted from the genotype.

We are grateful to F Langlet and C Souleyan for their technical assistance. We thank Dr B Lecolier for the fetal serum analysis and $\operatorname{Dr} C$ Horn for critical reviewing of the manuscript.

1 Conley ME, Bechwith JB, Mancer JFK, Tenckhoff L. The spectrum of the DiGeorge syndrome. $f$ Pediatr 1979;94:883-90.

2 De la Chapelle A, Herva R, Koivisto M, Aula P. A deletion in chromosome 22 can cause DiGeorge syndrome. Hum Genet 1981;57:253-6.

3 Greenberg F, Elder FFB, Haffner P, Northrup H, Ledbetter $\mathrm{DH}$. Cytogenetic findings in a prospective series of patients DH. Cytogenetic findings in a prospective series of patients 4 with DiGeorge anomaly. Am f Hum Genet 1988;43:605-11. 4 Scambler PJ, Carey AH, Wyse RKH, et al. Microdeletions
within 22q11 associated with sporadic and familial within 22q11 associated with sporadic and

5 Driscoll DA, Budarf ML, Emanuel BS. A genetic etiology for DiGeorge syndrome: consistent deletions and microdeletions of 22q11. Am $₹$ Hum Genet 1992;50:924-33.

6 Carey AH, Kelly D, Halford S, et al. Molecular genetic study of the frequency of monosomy $22 \mathrm{q} 11$ in DiGeorge syndrome. Am $\mathcal{Y}$ Hum Genet 1992;51:964-70.

7 Wilson DJ, Burn J, Scambler PJ, Goodship J. DiGeorge syndrome: part of CATCH 22. $\mathcal{F}$ Med Genet 1993;30:8526.

8 Glover 257-8.

Wandegent J, Collins $C$, et al. Fluorescence in situ hybridization with human chromosome-specific libraries: detection of trisomy 21 and translocations of chromosome 4. Proc Natl Acad Sci USA 1988;9:138-42.

10 Van Hemel JO, Schaap C, Van Opstal D, Mulder MF, Niermeijer MF, Meijers JHC. Recurrence of DiGeorge syndrome: prenatal detection by FISH of a molecular 22q11 deletion. 7 Med Genet 1995;32:657-8.

11 Puder KS, Humes RA, Gold RL, Bawle EV, Goyert GL. The genetic implication for preceding generations of the prenatal diagnosis of interrupted aortic arch in association prenatal diagnosis of interrupted aortic arch in association
with unsuspected DiGeorge anomaly. Am 7 Obstet Gynecol with unsuspected

12 Davidson A, Khandelawel M, Punnett HH. Prenatal diagnosis of the 22q11 deletion syndrome. Prenat Diagn 1997;17:380-3.

13 Van Mierop LHS, Kutsche LK. Cardiovascular anomalies in DiGeorge syndrome and importance of neural crest as a possible pathogenic factor. Am $\mathcal{f}$ Cardiol $1986 ; 58: 133-7$.

14 Amati F, Mari A, Digilio MC, et al. 22q11 deletions in isolated and syndromic patients with tetralogy of Fallot. Hum Genet 1995;95:479-82.

15 Momma K, Kondo C, Matsuoka R. Tetralogy of Fallot with pulmonary atresia associated with chromosome 22q11 pulmonary atresia associated with chrom
deletion. $₹ \mathrm{Am}$ Coll Cardiol 1996;1:198-202.

16 Lindsay EA, Greenberg F, Shaffer LG, Shapira SK, Scambler PJ, Baldini A. Submicroscopic deletions at 22q11.2: variability of the clinical picture. Am $\mathcal{F}$ Med Genet 1995;56:191-7.

17 Demczuk S, Levy A, Aubry M, et al. Excess of deletions of maternal origin in the DiGeorge/velo-cardio-facial syndromes. A study of 22 new patients and review of the literature. Hum Genet 1995;96:9-13.

18 Halford S, Lindsay EA, Nayudu M, Carey AH, Baldini A, Scambler PJ. Low-copy-number repeats flank the DiGeorge/velo-cardio-facial syndrome loci at $22 \mathrm{q} 11$. Hum Mol Genet 1993;2:191-6. 